University of Massachusetts Amherst

ScholarWorks@UMass Amherst

Physics Department Faculty Publication Series

Physics

1998

\title{
Search for physics beyond the standard model via a polarization-asymmetry correlation experiment on In-107
}

B Vereecke

J Camps

N Severijns

J Deutsch

T Otto

See next page for additional authors

Follow this and additional works at: https://scholarworks.umass.edu/physics_faculty_pubs

Part of the Physical Sciences and Mathematics Commons

\section{Recommended Citation}

Vereecke, B; Camps, J; Severijns, N; Deutsch, J; Otto, T; Govaerts, J; Brown, BA; Holstein, B; Kirchner, R; Naviliat-Cuncic, O; Prieels, R; Quin, PA; Thomas, E; De Moor, P; Schuurmans, P; Van Geert, A; and Vanneste, L, "Search for physics beyond the standard model via a polarization-asymmetry correlation experiment on In-107" (1998). ACTA PHYSICA POLONICA B. 337.

Retrieved from https://scholarworks.umass.edu/physics_faculty_pubs/337 
Authors

B Vereecke, J Camps, N Severijns, J Deutsch, T Otto, J Govaerts, BA Brown, B Holstein, R Kirchner, O

Naviliat-Cuncic, R Prieels, PA Quin, E Thomas, P De Moor, P Schuurmans, A Van Geert, and L Vanneste 


\title{
SEARCH FOR PHYSICS BEYOND THE STANDARD MODEL VIA A POLARIZATION-ASYMMETRY CORRELATION EXPERIMENT ON ${ }^{107} \mathrm{In}^{*}$
}

\author{
B. Vereecke ${ }^{\mathrm{a}}$, J. Camps ${ }^{\mathrm{a}}$, N. Severiuns ${ }^{\mathrm{a}}$, J. Deutsch $^{\mathrm{b}}$, T. Otto ${ }^{\mathrm{b}}$, \\ J. Govaerts ${ }^{b}$, B.A. Brown ${ }^{\mathrm{c}}$, B. Holstein ${ }^{\mathrm{d}}$, R. Kirchner ${ }^{\mathrm{e}}$, \\ O. Naviliat-Cuncic ${ }^{\mathrm{f}}$, R. PRIEels ${ }^{\mathrm{b}}$,

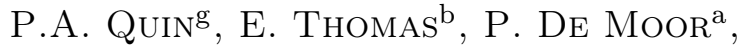 \\ P. Schunrmans ${ }^{\mathrm{a}}$, A. VAn Geert ${ }^{\mathrm{a}}$ And L. VAnneste ${ }^{\mathrm{a}}$

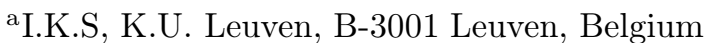 \\ ${ }^{b}$ I.P.N. U.C. de Louvain, B-1348 Louvain-la-Neuve, Belgium \\ ${ }^{\mathrm{c}}$ Department of Physics and Astronomy, Michigan State University \\ East Lansing, MI 48824, USA \\ ${ }^{\mathrm{d}}$ Department of Physics and Astronomy, University of Massachusetts \\ Amherst, MA 01002, USA \\ ${ }^{\text {e}}$ G.S.I. Darmstadt, D-64220 Darmstadt 11, Germany \\ ${ }^{\mathrm{f}}$ Institut für Teilchenphysik, E.T.H. Zürich \\ Hönggerberg, CH-8093 Zürich, Switzerland \\ g Department of Physics, University of Wisconsin \\ Madison, WI 53706, USA \\ (Received November 25, 1997)
}

We report on a new precision measurement of the longitudinal polarization of positrons emitted by polarized ${ }^{107}$ In nuclei. If interpreted in the framework of the manifest left-right symmetric model, preliminary results yield a lower limit of $303 \mathrm{GeV} / c^{2}$ for the mass of a possible, predominantly right-handed $\mathrm{W}$ gauge boson.

PACS numbers: 23.40. Bw, 27.60. $+\mathrm{j}$

\section{Introduction}

The standard electroweak model (SM) is very successful but has too many free parameters and ad-hoc assumptions to be accepted as the "ultimate" description of nature. Parity violation, in particular, is "explained"

\footnotetext{
* Presented at the XXV Mazurian Lakes School of Physics, Piaski, Poland, August 27-September 6, 1997.
} 
in the SM only by assuming a V-A structure in the Hamiltonian. The simplest extension that restores the parity-symmetry, is the manifest left-right symmetric (MLRS) model [1]. Here, an additional charged gauge boson $W_{2}$ is introduced which acquires a mass $m_{2}$ higher than the mass $m_{1}$ of the observed gauge boson $W_{1}$. The two gauge-bosons are proposed to couple with the same coupling constant $g$ to the left- and right-handed fermions according to the mixing scheme :

$$
W_{\mathrm{L}}=W_{1} \cos (\zeta)+W_{2} \sin (\zeta), W_{\mathrm{R}}=-W_{1} \sin (\zeta)+W_{2} \cos (\zeta)
$$

In more general left-right symmetric (LRS) models [2] distinct left/right coupling constants $g_{\mathrm{L}} / g_{\mathrm{R}}$ and Cabibbo-Kobayashi-Maskawa (CKM) matrix elements $V^{\mathrm{L}} / V^{\mathrm{R}}$ are introduced.

\section{Method and formalism}

We report on a new measurement of the longitudinal polarization $\mathrm{P}$ of positrons emitted by polarized ${ }^{107}$ In nuclei in which the result of our previous measurement [3] was improved significantly. This time, the longitudinal polarization of positrons emitted by polarized $\left(P^{-}\right)$and unpolarized nuclei $\left(P^{0}\right)$ were compared. The polarization-asymmetry correlation $R=P^{-} / P^{0}$ is potentially very sensitive to right-handed charged weak currents [4]. In the SM (i.e. $\delta=\zeta=0)$ the ratio of the two positron polarizations is given by :

$$
R_{0} \equiv \frac{P_{0}^{-}}{P_{0}^{0}}=\frac{\Theta^{-}}{\Theta^{0}} \frac{\beta^{2}-(\bar{\beta} \cdot \bar{J} A) \Psi}{\beta^{2}(1-\bar{\beta} \cdot \bar{J} A)}
$$

with $\beta=v / c$ the relative velocity of the positrons, $\bar{J}$ the polarization and A the $\beta$ asymmetry parameter. The functions $\Theta$ and $\Psi$ are solid angle integrals that take into account effects of the finite acceptance of our apparatus. The value $R_{0}$ can thus be obtained from a simultaneous measurement of the mean positron velocity and the emission asymmetry. Any deviation of the measured ratio from $R_{0}$ would either reflect contributions beyond the allowed approximation in nuclear $\beta$-decay (recoil effects), or indicate new physics beyond the SM. In the MLRS model and neglecting recoil effects, the ratio $\mathrm{R}$ can be written as:

$$
R=R_{0}\left[1-\frac{4(\bar{\beta} \cdot \bar{J} A) \Psi}{\beta^{2}-(\bar{\beta} \cdot \bar{J} A) \Psi}(\delta+\zeta)^{2}\right]
$$

with $\delta=\left(m_{1} / m_{2}\right)^{2}$. This ratio $\mathrm{R}$ thus depends on the mass of a $W_{2}$ boson. 


\section{Experimental set-up}

The $\beta$ decay of ${ }^{107} \operatorname{In}\left(t_{1 / 2}=32.4 \mathrm{~m}\right)$ is dominated by an allowed $9 / 2^{+} \rightarrow 7 / 2^{+}$pure GT transition with $\log f t=5.65$. A $10^{7}$ at $/ \mathrm{s}{ }^{107} \mathrm{In}$ beam from the LISOL separator coupled on-line to the CYCLONE cyclotron was implanted into a Fe foil inside the KOOL on-line low temperature nuclear orientation setup, where the ${ }^{107}$ In was polarized to $\approx 80 \%$ at a temperature of $\approx 11 \mathrm{mK}$. By raising the temperature to $1 \mathrm{~K}$ an unpolarised ${ }^{107} \mathrm{In}$ source was obtained. The positrons emitted by the ${ }^{107}$ In nuclei were energy selected with a magnetic spectrometer and transported to a positron polarimeter. This polarimeter is based on the method of time-resolved positronium hyperfine spectroscopy to determine the positron polarizations [5]. The technique relies on the fact that in a magnetic field the $m= \pm 1$ triplet (TR; $\tau \approx 129 n s$ ) substates of the Ps are not affected, while the singlet state and the $m=0$ triplet substate are perturbed by the field and mix, leading to a pseudo-singlet (PS; $\tau \leq 2.5 n s)$ and a pseudo-triplet $(\mathrm{PT} ; \tau \approx 7.5 n s)$ state. The population of the PS and PT states depends on the polarization $\mathrm{P}$ of the positrons, the magnetic field $\mathrm{B}$ and on the relative direction of $\bar{B}$ and $\bar{P}$. Comparing the decay intensities of the PT states (region between 2.5 and 30 $\mathrm{ns}$ ) for two opposite ( $\mathrm{p}$ and a) field directions of the polarimeter magnetic field, the polarization along the field axis can be determined. In figure 1 experimental Ps decay spectra are plotted for both field directions. Also, the response function, obtained by replacing the $\mathrm{MgO}$ powder, in which the Ps was formed, by an $\mathrm{Al}$ pellet in which all positrons annihilate directly, is shown.

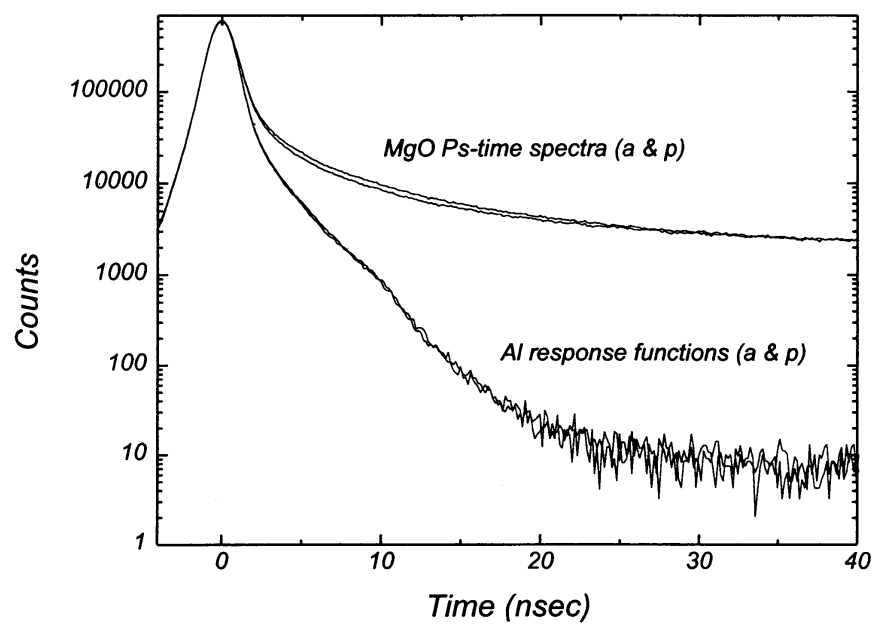

Fig. 1. Experimental Ps decay spectrum and response function. 


\section{Analysis}

From the above it is clear that for this experiment, 3 quantities need to be determined experimentally : the polarization ratio $R=P^{-} / P^{0}$, the beta asymmetry $\bar{\beta} \cdot \bar{J} A$ and the positron velocity $\beta$. The latter was calculated on the basis of the momentum acceptance and calibration of the spectrometer. The beta asymmetry was obtained from the intensity ratio -after normalizing to the source intensity- of the triplet Ps state in the interval 80ns $<t<250 \mathrm{~ns}$ for polarized and unpolarized nuclei. The positron polarizations $P^{-}$and $P^{0}$ are determined from the PT region in the Ps decay spectrum.

The possible impact of systematic effects was determined by several calibration experiments. From measurements on a ${ }^{68} \mathrm{Ga}$ source, used with and without backing on a rotatable sourceholder, we found that about $6 \%$ of the count rate in the detector originates from scattered positrons. However, because scattering is mainly forward, the polarization-asymmetry correlation is preserved. Errors arising from small time shifts in the Ps decay spectra were taken into account by shifting the spectra.

Comparing the data from unpolarized nuclei in the different runs showed that no significant changes due to different source positions were present. Recoil effects were calculated using the formalism of Holstein [6], limiting ourselves to contributions up to order $1 / \mathrm{M}$, with $\mathrm{M}$ the mass off the daughter nucleus. We found that $\left(R / R_{0}\right)_{\text {recoil }}=0.9950(14)$ and all measurements were corrected for this effect. Finally, calculations to allow correction for the spin precession of the positrons are in progress. Estimates show this to induce a minor effect only.

\section{Results and discussion}

The preliminary, weighted average result of all 13 runs carried out is $R / R_{0}=0.9898(82)$. This value constitutes an improvement in precision of more than a factor three compared to the previous result [3]. If interpreted in the manifest left-right symmetric model it corresponds to a lower limit of $303 \mathrm{GeV} / \mathrm{c}^{2}(90 \%$ C.L.; $\zeta=0)$ for the mass of a charged $W_{\mathrm{R}}$ gauge boson with right-handed couplings. For comparison, the combined limit from all the nuclear $\beta$-decay experiments is $320 \mathrm{GeV} / \mathrm{c}^{2}$ (90\% C.L.). The mass limits from $\mu$-decay $\left(m_{2}>482 \mathrm{GeV} / \mathrm{c}^{2}, 90 \%\right.$ C.L.) [7] and direct searches $\left(m_{2}>\right.$ $720 \mathrm{GeV} / \mathrm{c}^{2}, 95 \%$ C.L) [8] are superior if interpreted in the MLRS model. In more general LRS models our result, however, is complementary to results from other sectors in the weak interaction, as was shown in [9]. Note that collider searches loose their sensitivity to a heavy $\mathrm{W}$ boson for masses below $100 \mathrm{GeV} / \mathrm{c}^{2}$. 


\section{REFERENCES}

[1] J.C. Pati, A. Salam, Phys. Rev. D10, 275 (1974); M.A.B. Beg et al., Phys. Rev. Lett. 38, 1252 (1977).

[2] P. Langacker, U. Sankar, Phys. Rev. D45, 278 (1992).

[3] N. Severijns et al., Phys. Rev. Lett. 704047 (1993); 73, 611E (1994).

[4] P.A. Quin, T.A. Girard, Phys. Lett. A229, 29 (1989).

[5] R. Prieels, in Proceedings of the 24th Rencontre de Moriond, January 1989, Eds. Frontières, Gif-sur-Yvette, 1989, p. 287.

[6] B.R. Holstein, Rev. Mod. Phys. 46, 789 (1974); 48, 673E (1976).

[7] A. Jodidio et al., Phys. Rev. D34, 1967 (1986); Phys. Rev. D37, 237E (1988).

[8] S. Abachi et al., Phys. Rev. Lett. 76, 3271 (1996).

[9] P.A. Quin et al., in Proceedings of the Conference on Particles and Nuclei (PANIC), Williamsburg, USA, May 1996, to be published. 